\title{
Non-alcoholic liver disease and childhood obesity
}

M Shaunak ${ }^{(1,4)}$, CD Byrne ${ }^{(2,3)}$, N Davis ${ }^{(3,4)}$, P Afolabi ${ }^{(2,3)}$, SN Faust ${ }^{(1,2,3)}$, JH Davies ${ }^{(3,4)}$

(1) NIHR Southampton Clinical Research Facility, University Hospital Southampton NHS Foundation Trust, University Hospital Southampton NHS Foundation Trust, Southampton, UK

(2) NIHR Southampton Biomedical Research Centre, University Hospital Southampton NHS Foundation Trust, Southampton, UK

(3) University of Southampton Faculty of Medicine and Institute for Life Sciences, University of Southampton, UK

(4) Department of Paediatric Endocrinology, Southampton Children's Hospital, University Hospital Southampton NHS Foundation Trust, Southampton, UK

Corresponding Author: Dr Justin Davies, Department of Paediatric Endocrinology, University Hospital Southampton NHS Foundation Trust, Southampton SO16 6YD

Email: justin.davies@uhs.nhs.uk

Financial disclosure: nil

Funding source: nil

Competing interest: none declared

Authors Contribution: Dr Shaunak drafted the initial manuscript and revised the manuscript. Professors Byrne and Faust and Drs Davies, Davis and Afolabi reviewed and revised the manuscript. All authors approved the final manuscript as submitted.

Word count: 3062 


\section{ABSTRACT}

Non-alcoholic fatty liver disease (NAFLD) in children and adolescents has an estimated prevalence of $36.1 \%$ in the context of obesity. This figure is anticipated to increase in conjunction with the global obesity epidemic. Worryingly, NAFLD in childhood persisting into adulthood is likely to be harmful, contributing to significant hepatic and extra-hepatic morbidity. Early disease detection is required, although the optimum timing, frequency and mode of screening remains undetermined. Whilst the efficacy of several medications, antioxidants, fatty acid supplements and probiotics have been investigated in children, healthy eating and physical activity, remain the only prevention and treatment strategies for paediatric NAFLD. This short review discusses the epidemiology, diagnosis, pathogenesis and management of NAFLD in childhood obesity. 


\section{INTRODUCTION}

The term "non-alcoholic fatty liver disease" can either be used to summarise a histopathological spectrum of liver disease, which progresses from steatosis to nonalcoholic steatohepatitis (NASH), or it can be used to describe early benign disease prior to the onset of NASH.

In adults, NAFLD not only increases the risk of cirrhosis and hepatocellular carcinoma, but also contributes to the development of type 2 diabetes mellitus, cardiovascular disease and chronic kidney disease and is therefore increasingly considered to be a multi-system disease[1]. In children and adolescents, NAFLD is similarly associated with significant extrahepatic morbidity[2-4]. Although the long-term consequences of paediatric NAFLD remain largely unknown due to the lack of high-quality longitudinal data, it seems reasonable to assume that NAFLD in childhood persisting into adulthood is likely to be a harmful condition.

Adiposity, as defined by body mass index (BMI), is an important risk factor for NAFLD, with prospective studies in children demonstrating a clear association between adiposity gain in childhood and adverse liver outcomes in adolescence[5]. Adiposity-related risk is predominantly attributed to the development of insulin resistance and adipose tissue dysfunction in these individuals. The prevalence of NAFLD in children and adolescents is therefore anticipated to increase in conjunction with the global obesity epidemic that currently affects millions of young people worldwide.

This short review will discuss: a) the epidemiology of childhood NAFLD, including the effects of ethnicity; b) the diagnosis of childhood NAFLD including invasive and non-invasive testing; c) risk factors and pathogenesis of NAFLD in childhood; and d) the management and treatment of NAFLD in childhood. 


\section{a) The epidemiology of childhood NAFLD, including the effects of ethnicity}

It has proved difficult to achieve a precise estimate of the prevalence of NAFLD in children and adolescents. Differences in study design and the methodology used to diagnose NAFLD, plus the changing prevalence and inconsistent definition of obesity in childhood, have all contributed to uncertainty regarding the exact prevalence of NAFLD in this age group. Many studies have defined NAFLD on the basis of screening biochemistry and/or imaging, rather than liver biopsy, which is the generally accepted "gold standard" diagnostic investigation[6,7].

Anderson et al (2015) conducted a systematic review and meta-analysis to estimate the prevalence of NAFLD in children and adolescents, aged $\geq 1$ and $\leq 19$ years[8]. Using data from nine general population studies, the authors reported NAFLD prevalence estimates of $2.3 \%$ (95\% Cl: $1.5 \%$ to $3.6 \%$ ) in normal weight individuals, $12.5 \%$ (95\% Cl: $9.2 \%$ to $16.7 \%$ ) in overweight individuals and $36.1 \%$ (95\% Cl: $24.6 \%$ to $49.4 \%$ ) in obese individuals, with a higher prevalence reported in males compared to females[8].

The prevalence of NAFLD in obese populations shows geographical variation, partly reflecting the genetic contribution to NAFLD aetiology[8]. Nobili et al (2019) mapped the prevalence of paediatric NAFLD, reporting the highest prevalence in Central America and the Middle East, with figures of $42.5 \%$ in children aged 8 to 11 years determined by alanine aminotransferase (ALT) in Mexico and 16.9\% in children aged 6 to 19 years determined by ultrasound in Iran[3].

\section{b) The diagnosis of childhood NAFLD including invasive and non-invasive testing}

\section{Screening}

The European and North American Societies for Paediatric Gastroenterology, Hepatology and Nutrition (ESPGHAN and NASPGHAN) have published clinical guidance for the diagnosis of paediatric NAFLD[6,7]. Some children and adolescents with NAFLD present with non- 
specific symptoms, including generalised abdominal discomfort and fatigue, whilst others are asymptomatic. ESPGHAN recommends performing screening liver function tests (LFTs) and ultrasonography in all children and adolescents with obesity (BMI $>95^{\text {th }}$ percentile), prior to stratifying further management according to age, history and physical examination[6]. By contrast, NASPGHAN recommends measuring ALT from the age of 9 years in all overweight $\left(\mathrm{BMI} \geq 85^{\text {th }}\right.$ and $<94^{\text {th }}$ percentile) and obese $\left(\mathrm{BMI} \geq 95^{\text {th }}\right.$ percentile) children, provided the former have additional risk factors for NAFLD[7]. Both recommend re-screening should initial results return normal, although the optimum timing and frequency of re-screening remain undetermined[6,7].

Despite the pragmatic argument for using ALT as a screening investigation, clinicians should be aware that ALT has a low sensitivity for NAFLD and values do not correlate with ultrasound or biopsy findings - a 'normal' ALT value may therefore be falsely reassuring $[9,10]$. Age- and sex-related reference ranges of ALT have been developed in children. These should be considered when interpreting ALT results rather than necessarily using laboratory reported upper limits of normal[11,12]. Increasingly refined paediatric NAFLD plasma screening panels are in development, combining ALT with other metabolic data, to improve screening specificity[13].

It is also important to note that ultrasound has been shown to have a high sensitivity and specificity for moderate to severe steatosis in children and young people, however mild steatosis may not be detected[9]. By contrast, MRI estimated hepatic proton density fat fraction (PDFF) allows quantification of steatosis severity. MRI PDFF values show significant correlation with histological steatosis grade and may be more useful than ultrasound in defining early disease[14-16].

\section{$\underline{\text { Differential diagnoses }}$}

NAFLD is a diagnosis of exclusion. Following the identification of an elevated ALT and/or hyper-echogenicity on ultrasound, clinicians should consider genetic, metabolic and systemic causes of fatty liver disease, particularly in young children and those with very high 
or chronically elevated ALT values. Table 1 lists important secondary causes of hepatic steatosis in children and adolescents[6,7].

Table 1: Secondary causes of hepatic steatosis

\begin{tabular}{|c|c|}
\hline \multicolumn{2}{|l|}{ Causes } \\
\hline Endocrine & $\begin{array}{l}\text { Growth hormone deficiency } \\
\text { Hypothyroidism } \\
\text { Hypogonadism } \\
\text { Polycystic ovarian syndrome } \\
\text { Type } 1 \text { diabetes mellitus }\end{array}$ \\
\hline Hepatic & $\begin{array}{l}\text { Wilson's disease } \\
\text { Haemochromatosis } \\
\text { Alpha } 1 \text { anti-trypsin deficiency }\end{array}$ \\
\hline Gastrointestinal & $\begin{array}{l}\text { Coeliac disease } \\
\text { Inflammatory bowel disease }\end{array}$ \\
\hline Metabolic & $\begin{array}{l}\text { Inborn error of metabolism } \\
\text { Disorders of lipid and lipoprotein metabolism } \\
\text { Lipodystrophies }\end{array}$ \\
\hline Renal & Nephrotic syndrome \\
\hline Genetic & $\begin{array}{l}\text { Alstrom syndrome } \\
\text { Prader Willi syndrome } \\
\text { Bardet-Biedl syndrome }\end{array}$ \\
\hline Respiratory & $\begin{array}{l}\text { Cystic fibrosis } \\
\text { Obstructive sleep apnoea }\end{array}$ \\
\hline Infection & $\begin{array}{l}\text { Hepatitis C } \\
\text { HIV } \\
\text { Bacterial overgrowth }\end{array}$ \\
\hline Medications & $\begin{array}{l}\text { Corticosteroids } \\
\text { Oestrogens } \\
\text { Amiodarone } \\
\text { Sodium valproate } \\
\text { Nifedipine } \\
\text { Diltiazem } \\
\text { Methotrexate } \\
\text { Antiretrovirals } \\
\text { TPN }\end{array}$ \\
\hline Other & $\begin{array}{l}\text { Alcohol misuse } \\
\text { Rapid weight loss } \\
\text { Anorexia nervosa } \\
\text { Protein energy malnutrition }\end{array}$ \\
\hline
\end{tabular}

$\underline{\text { Invasive evaluation of hepatic fibrosis }}$ 
Liver biopsy remains the gold standard diagnostic investigation, as it is currently the only test that can reliably differentiate steatosis from NASH and exclude potentially treatable liver disease.

Histologically, NAFLD is characterised by steatosis affecting more than $5 \%$ of hepatocytes - a definition developed from adult data[17]. Steatosis with mild inflammation can progress to $\mathrm{NASH}$, with increasing inflammation, hepatocyte injury and fibrosis. Worryingly, in children and adolescents, NASH rather than steatosis is more commonly identified by the time of liver biopsy[18].

The histological pattern of NASH differs depending on age, gender and ethnicity[19]. Two types of paediatric NASH have been described, although features may overlap. Type 1 or 'adult type' NASH is characterised by steatosis, inflammation and hepatocyte injury, demonstrated by ballooning and the variable presence of Mallory-Denk bodies, predominantly affecting 'zone 3' hepatocytes surrounding central veins[20]. In Type 2 NASH, steatosis is often more pronounced in 'zone 1' hepatocytes, which surround portal tracts[20]. Inflammation is generally mild and evidence of hepatocyte injury is difficult to identify[20].

An estimated $30-40 \%$ of adults with NAFLD develop NASH and $40-50 \%$ of these develop hepatic fibrosis, which can progress to cirrhosis and end-stage liver failure, an increasingly common indication for transplantation[1]. Although the natural history of paediatric NAFLD remains largely unknown, available evidence indicates that severe fibrosis and cirrhosis are potential consequences of disease starting in childhood and worryingly, disease progression can be rapid[2]. In adults, NASH is also a well-documented risk factor for hepatocellular carcinoma[1]. Although rare, cases of hepatocellular carcinoma have been reported in paediatric NAFLD[2].

Despite the risk posed by NASH, a presumptive diagnosis of paediatric NAFLD is often made in the absence of other causes of fatty liver, largely due to the limitations of liver biopsy specifically the invasive nature of the procedure, the risk of sampling error and the high cost 
involved[20]. There is no current consensus on the indications for liver biopsy in children and adolescents[6,7].

\section{Non-invasive evaluation of hepatic fibrosis}

There is therefore a need for accurate non-invasive tests for liver fibrosis in children and young people. Certain hepatic fibrosis scores (aspartate aminotransferase (AST)/ALT ratio, AST/ platelet ratio index (APRI), NAFLD fibrosis score (NFS) and FIB-4 index), developed for the adult population, have not been validated in paediatric studies of biopsy-proven NAFLD[21].

The enhanced liver fibrosis (ELF) test has been shown to reliably reflect liver fibrosis in paediatric NAFLD[22]. However, this test is reported to be expensive and not widely available outside the United Kingdom[23]. One of the biomarkers used in the ELF test, P3NP, has recently been shown to accurately predict liver fibrosis severity in children[23]. This test, together with promising paediatric fibrosis prediction scores (PNFI and PNFS), await external validation[22,24]. ${ }^{13} \mathrm{C}$-liver function breath tests represent a novel non-invasive approach for monitoring NAFLD progression in adults, although their application in children with NAFLD is yet to be ascertained[25,26].

In terms of imaging techniques, ultrasound is unable to differentiate between steatosis and NASH[9]. Transient elastography and magnetic resonance elastography (MRE) are both used for the non-invasive assessment of liver fibrosis in the adult population, but their usefulness in children and adolescents with fatty liver disease remains under evaluation[2729].

\section{Other tests}

NAFLD is increasingly considered to be an additional component of metabolic syndrome, although the diagnostic criteria for metabolic syndrome in children and adolescents remain inconsistently defined[30]. Metabolic investigations, including fasting plasma glucose, glycosylated haemoglobin A1c (HbA1c) and lipid profile (total cholesterol, high-density 
lipoprotein, low-density lipoprotein, triglycerides), are therefore indicated to screen for comorbidities associated with NAFLD such as impaired fasting glycaemia, type 2 diabetes mellitus and dyslipidaemia[6,7].

\section{c) Risk factors and pathogenesis of NAFLD in childhood}

\section{i) $\quad$ Risk factors (Table 2)}

Age and sex:

Males have a higher prevalence of NAFLD than females, both before and during puberty[31]. Hormonal changes accompanying puberty contribute to NAFLD pathogenesis. Increases in insulin-like growth factor 1 (IGF-1) protect against hepatic steatosis[32]. Similarly, oestrogens are thought to have an anti-inflammatory and insulin sensitising role, as well as influencing body composition, favouring non-visceral rather than visceral adipose tissue distribution[31,32]. However, the persistence of pre-pubertal sex differences in NAFLD prevalence through adolescence, highlights the influence of other factors, not just pubertal factors, on pathophysiology.

\section{Lifestyle factors:}

BMI remains an important risk factor for NAFLD in children and young people, with adipose tissue dysfunction contributing to NAFLD pathogenesis[33,34]. Unsurprisingly, greater energy intake in childhood and early adolescence is associated with an increased risk of NAFLD[8]. As well as overnutrition, the type of nutrition is important, with fructose, transand saturated fatty acids playing a role in disease aetiology[35]. Risk is compounded by obesity-related complications, such as obstructive sleep apnoea (OSA), where chronic intermittent hypoxia is thought to exacerbate liver oxidative stress, through reactive oxygen species (ROS) generation, thereby promoting progression of NAFLD[36]. Importantly, NAFLD does not occur in all individuals who are overweight or obese and can occur in lean individuals[37, 38]. 
Metabolic factors:

Insulin resistance, secondary to lifestyle, hormonal or genetic factors, is central to the development of NASH. Children with NAFLD are more likely to have biochemical evidence of insulin resistance, with higher fasting blood glucose, $\mathrm{HbA1c}$, insulin and homeostatic model of assessment of insulin resistance (HOMA-IR) levels[34]. Hepatic mitochondrial dysfunction could also play a significant role in disease progression to $\mathrm{NASH}$, but is yet to be confirmed in paediatric NAFLD $[39,40]$.

Gut-liver axis:

Studies of the faecal microbiome have identified intestinal dysbiosis in children and adolescents with NAFLD[41,42]. Altered gut flora is thought to result in increased free fatty acid production and changes to small bowel permeability. This leads to greater absorption of these free fatty acids, contributing to lipotoxicity and inflammation[33,42].

Genetic factors:

Siblings and parents of children with NAFLD are more likely to have fatty livers [43]. This may represent the influence of environment, however a genetic susceptibility to NAFLD aetiology is well described $[2,3,44]$. Reported genetic risk factors for NAFLD include PNPLA3, TM6SF2, GCKR and MBOAT7 variants[3,45]. The PNPLA3 variant encodes the protein I148M, which is strongly associated with increased hepatic fat levels and inflammation. This predisposing variant is more commonly found in Hispanic individuals compared to other ethnic groups, explaining their increased susceptibility to NAFLD[46].

Epigenetic factors:

The maternal environment is increasingly thought to program predisposition to NAFLD in offspring through epigenetic changes[47]. A history of maternal obesity is associated with increased liver fat in adolescence, independent of adiposity[48]. Alterations to the gut microbiome of infants born to obese mothers is one of a variety of causal mechanisms 
proposed $[47,49,50]$. Extremes of birth weight, compared to normal birth weight, are associated with more severe liver disease at biopsy, implicating maternal and uterine factors in pathogenesis[51]. Animal models also highlight the potential importance of paternally inherited epigenetic modifications in offspring susceptibility to fatty liver[52].

Environmental factors:

Animal studies suggest that environmental exposure to endocrine disrupting chemicals (EDCs) may contribute to the development and progression of NAFLD[53].

Table 2: Risk factors for paediatric NAFLD

\begin{tabular}{|l|l|}
\hline Modifiable risk factor & Non-modifiable risk factor \\
\hline BMI & Age \\
Diet & Sex \\
Physical activity & Ethnicity \\
Vitamin D deficiency & Birth weight \\
OSA & Genetic pre-disposition \\
& Family history of obesity or NAFLD \\
\hline
\end{tabular}

\section{ii) Pathogenesis}

The 'multiple hit' hypothesis describes NAFLD pathogenesis, where a number of insults are thought to act together to induce NAFLD development and progression in genetically predisposed or high-risk individuals (Figure 1)[32].

\section{Figure 1: The pathogenesis of NAFLD}

In obesity, pro-inflammatory cytokines released from hypertrophic adipose tissue, contribute to systemic insulin resistance[32]. Adipose tissue dysfunction also alters the balance of secreted adipokines, specifically the hormones leptin and adiponectin, which are thought to have pro- and anti-inflammatory roles respectively[33]. Uninhibited adipose tissue lipolysis, together with dietary intake and intestinal dysbiosis, leads to increased levels of free fatty acids within the circulation. This results in a hepatic influx of free fatty 
acids. Hepatic de novo lipogenesis also adds to the accumulation of these free fatty acids, which undergo oxidation, storage as triglyceride or excretion via very low-density lipoprotein (VLDL)[32]. Resultant lipotoxicity occurs when hepatocellular metabolic capacity is exceeded. Mitochondrial dysfunction leads to the overproduction of ROS and endoplasmic reticulum (ER) stress results in the accumulation of unfolded proteins. The combined effect accelerates progression to NASH with hepatocyte inflammation, apoptosis and fibrosis[33].

\section{d) The management and treatment of NAFLD in childhood.}

i) Management (Table 3)

\section{Lifestyle interventions}

In children and young people, biomarkers of NAFLD have been shown to improve following weight loss secondary to lifestyle modifications[54]. However, lifestyle interventions, such as low-calorie diets or exercise programmes, that do not culminate in weight loss, also appear to be beneficial[54]. There is no compelling high-quality evidence to recommend one nutritional intervention over another. Whilst low fructose, low fat and low glycaemic index diets have shown limited results, preliminary data on the efficacy of a low free sugar diet is promising[55]. General healthy eating advice and physical activity is currently recommended to promote weight loss in paediatric NAFLD, although the amount of weight loss necessary remains unclear[54,56,57].

\section{Management of co-morbidities and risk factors}

Management of extra-hepatic co-morbidities, including insulin resistance and dyslipidaemia, and modification of risk factors known to compound paediatric NAFLD are important. Treating children with OSA has been shown to improve NAFLD severity and optimisation of Vitamin D levels may minimise fibrosis[58,59]. Given the frequent association with psychological morbidity, the provision of psychological support as part of multi-disciplinary management may contribute to improved clinical outcomes[60]. 


\section{ii) $\quad \underline{\text { Treatment }}$}

The efficacy of several medications, antioxidants, fatty acid supplements and probiotics have been investigated in children. Whilst pioglitazone has been recommended for advanced liver fibrosis in adults, there is currently no pharmacological treatment of proven benefit for paediatric NAFLD.

\section{Metformin}

Metformin improves insulin resistance and may reduce steatosis on ultrasound[54]. The TONIC trial compared placebo, metformin and Vitamin E, an antioxidant, in children with histologically confirmed NAFLD, using sustained reduction in ALT as their primary outcome[61]. Both metformin and Vitamin E were no better than placebo with regards the primary outcome, but these groups did show evidence of reduced hepatocyte injury on biopsy[61]. In addition, the role of metformin as a weight loss adjunct in these children and young people remains under evaluation, with only a very modest, short-term reduction in BMI seen on meta-analysis[62].

\section{Anti-oxidants}

Adjuvant Vitamin E therapy has been shown to be efficacious in adults with NAFLD, however a similar significant effect has not been demonstrated on meta-analysis in paediatric patients[63]. Vitamin E therefore remains unlicensed for this indication in the United Kingdom, although may be considered for children with advanced liver fibrosis in tertiary care settings[64].

Vitamin E in combination with another antioxidant, hydroxytyrosol (HXT), may have potential, improving oxidative stress parameters, insulin resistance and steatosis in children with NAFLD[65]. Cysteamine bitartrate, may also have therapeutic benefit[66].

Polyunsaturated fatty acids 
Polyunsaturated fatty acids (PUFAs), including omega-3 fatty acids, are thought to have a beneficial effect on hepatic lipid accumulation[67,68]. However, a systematic review of trials involving PUFAs in paediatric NAFLD caveated benefit with variation in formulation and dosing between trials[54]. Further research is warranted.

\section{Probiotics}

Probiotics, such as bifidobacteria, are under investigation appearing to have a protective role in the development of NAFLD $[54,69]$.

\section{Surgical intervention}

The number of children and young people undergoing bariatric surgery is increasing worldwide[70]. Although bariatric surgery can improve histological findings in paediatric $\mathrm{NASH}$, surgical intervention remains controversial in all but exceptional clinical situations[71].

\section{Current clinical trials}

Losartan is currently being compared to placebo in 8 to 17 year olds with histological evidence of NAFLD. Losartan is thought to reduce plasminogen activator inhibitor-1 (PAI-1), which is elevated in children with NAFLD and associated with increased disease severity[72]. Another clinical trial is evaluating two doses of elafibranor ( 80 and 120 milligrams) in 8 to 17 year olds with histological evidence of NASH. Elafibranor improves insulin sensitivity and has been shown to induce resolution of NASH in adult patients[73].

Clinical trials involving supplementation with turmeric preparations, amino acid formulations and non-caloric sweetener and dietary interventions involving sugar reduction, a 5:2 diet and a low-carb-high-fat diet in paediatric NAFLD are also underway.

Table 3: The management of paediatric NAFLD 


\begin{tabular}{|l|l|}
\hline Non-pharmacological management & Pharmacological management \\
\hline Dietary advice & Vitamin D \\
Increased physical activity & Metformin \\
Psychological support & Lipid lowering agents \\
Respiratory support for OSA & Blood pressure lowering agents \\
& \\
\hline
\end{tabular}

\section{CONCLUSION}

The true prevalence of paediatric NAFLD in obesity will remain unknown, until the field adopts a unified pragmatic approach to defining disease, to minimise study heterogeneity. With international disease registries such as the European Paediatric Non-Alcoholic Fatty Liver Disease Register (EU-PNAFLD), longitudinal data on disease progression will allow optimisation of screening practice and accurate estimation of the health and socioeconomic burden of NAFLD starting in childhood. The development of readily available non-invasive methods for evaluating hepatic fibrosis, will aid timely investigation and inform counselling discussions relating to hepatic and extra-hepatic risk. Growing knowledge of the complex pathophysiology of NAFLD continues to reveal potential therapeutic targets. However, whilst the hunt for therapeutic agents continues, healthy eating and physical activity, remain the only prevention and treatment strategies for paediatric NAFLD. 


\section{REFERENCES}

1 Byrne CD, Targher G. NAFLD: a multisystem disease. J Hepatol. 2015; 62: S47-64.

2 Goyal NP, Schwimmer JB. The Progression and Natural History of Pediatric Nonalcoholic Fatty Liver Disease. Clin Liver Dis. 2016; 20(2): 325-338.

3 Nobili V, Alisi A, Valenti L, et al. NAFLD in children: new genes, new diagnostic modalities and new drugs. Nat Rev Gastroenterol Hepatol. 2019; 16(9): 517-530.

4 Pacifico L, Perla FM, Roggini M, et al. A Systematic Review of NAFLD-Associated Extrahepatic Disorders in Youths. J Clin Med. 2019; 8(6).

5 Anderson EL, Howe LD, Fraser A, et al. Weight trajectories through infancy and childhood and risk of non-alcoholic fatty liver disease in adolescence: the ALSPAC study. J Hepatol. 2014; 61(3): 626-632.

6 Vajro P, Lenta S, Socha P, et al. Diagnosis of nonalcoholic fatty liver disease in children and adolescents: position paper of the ESPGHAN Hepatology Committee. J Pediatr Gastroenterol Nutr. 2012; 54(5): 700-713.

7 Vos MB, Abrams SH, Barlow SE, et al. NASPGHAN Clinical Practice Guideline for the Diagnosis and Treatment of Nonalcoholic Fatty Liver Disease in Children. J Pediatr Gastroenterol Nutr. 2017; 64: 319-334.

8 Anderson EL, Howe LD, Jones HE, et al. The Prevalence of Non-Alcoholic Fatty Liver Disease in Children and Adolescents: A Systematic Review and Meta-Analysis. PLoS ONE. 2015; 10(10): e0140908.

9 Shannon A, Alkhouri N, Carter-Kent C, et al. Ultrasonographic quantitative estimation of hepatic steatosis in children with NAFLD. J Pediatr Gastroenterol Nutr. 2011; 53(2): 190-195.

10 Johansen MJ, Gade J, Stender S, et al. The Effect of Overweight and Obesity on Liver Biochemical Markers in Children and Adolescents. J Clin Endocrinol Metab. 2020; 105(2): 430-442.

11 England K, Thorne C, Pembrey L, et al. Age- and sex-related reference ranges of alanine aminotransferase levels in children: European paediatric HCV network. J Pediatr Gastroenterol Nutr. 2009; 49(1): 71-77.

12 Colantonio DA, Kyriakopoulou L, Chan MK, et al. Closing the Gaps in Pediatric Laboratory Reference Intervals: A CALIPER Database of 40 Biochemical Markers in a Healthy and Multiethnic Population of Children. Clin Chem. 2012; 58(5): 854-868.

13 Khusial RD, Cioffi CE, Caltharp SA, et al. Development of a Plasma Screening Panel for Pediatric Nonalcoholic Fatty Liver Disease Using Metabolomics. Hepatol Commun. 2019; 3(10): 1311-1321. 
14 Schwimmer JB, Middleton MS, Behling C, et al. Magnetic resonance imaging and liver histology as biomarkers of hepatic steatosis in children with nonalcoholic fatty liver disease. Hepatology. 2015; 61(6): 1887-1895.

15 Manning $\mathrm{P}$, Murphy $\mathrm{P}$, Wang $\mathrm{K}$, et al. Liver histology and diffusion-weighted $\mathrm{MRI}$ in children with nonalcoholic fatty liver disease: A MAGNET study. J Magn Reson Imaging. 2017; 46(4): 1149-1158.

16 Middleton MS, Van Natta ML, Heba ER, et al. Diagnostic accuracy of magnetic resonance imaging hepatic proton density fat fraction in pediatric nonalcoholic fatty liver disease. Hepatology. 2018; 67(3): 858-872.

17 Schild MH, Guy CD. Nonalcoholic Steatohepatitis: Histopathology basics within a broader context. Surg Pathol Clin. 2018; 11(2): 267-285.

18 Schwimmer JB, Behling C, Newbury R, et al. Histopathology of pediatric nonalcoholic fatty liver disease. Hepatology. 2005; 42(3): 641-649.

19 Africa JA, Behling CA, Brunt EM, et al. In Children With Nonalcoholic Fatty Liver Disease, Zone 1 Steatosis Is Associated With Advanced Fibrosis. Clin Gastroenterol Hepatol. 2018; 16(3): 438-446.e1.

20 Kleiner DE, Makhlouf HR. Histology of Nonalcoholic Fatty Liver Disease and Nonalcoholic Steatohepatitis in Adults and Children. Clin Liver Dis. 2016; 20(2): 293-312.

21 Mansoor S, Yerian L, Kohli R, et al. The evaluation of hepatic fibrosis scores in children with nonalcoholic fatty liver disease. Dig Dis Sci. 2015; 60(5): 1440-1447.

22 Nobili V, Alisi A, Vania A, et al. The pediatric NAFLD fibrosis index: a predictor of liver fibrosis in children with non-alcoholic fatty liver disease. BMC Med. 2009; 7: 21.

23 Mosca A, Comparcola D, Romito I, et al. Plasma N-terminal propeptide of type III procollagen accurately predicts liver fibrosis severity in children with non-alcoholic fatty liver disease. Liver Int. 2019; 39(12): 2317-2329.

24 Alkhouri N, Mansoor S, Giammaria P, et al. The development of the pediatric NAFLD fibrosis score (PNFS) to predict the presence of advanced fibrosis in children with nonalcoholic fatty liver disease. PLOS ONE. 2014; 9(8): e104558.

25 Portincasa P, Grattagliano I, Lauterburg BH, et al. Liver breath tests non-invasively predict higher stages of non-alcoholic steatohepatitis. Clin Sci (Lond). 2006; 111(2): 135-143.

26 Banasch M, Ellrichmann M, Tannapfel A, et al. The non-invasive (13)C-methionine breath test detects hepatic mitochondrial dysfunction as a marker of disease activity in nonalcoholic steatohepatitis. Eur J Med Res. 2011; 16(6): 258-264. 
27 Desai NK, Harney S, Raza R, et al. Comparison of Controlled Attenuation Parameter and Liver Biopsy to Assess Hepatic Steatosis in Pediatric Patients. J Pediatr. 2016; 173: 160164.e1.

28 Kwon YD, Ko KO, Lim JW, et al. Usefulness of Transient Elastography for Non-Invasive Diagnosis of Liver Fibrosis in Pediatric Non-Alcoholic Steatohepatitis. J Korean Med Sci. 2019; 34(23): e165.

29 Schwimmer JB, Behling C, Angeles JE, et al. Magnetic resonance elastography measured shear stiffness as a biomarker of fibrosis in pediatric nonalcoholic fatty liver disease. Hepatology. 2017; 66(5): 1474-1485.

30 Weihe P, Weihrauch-Blüher S. Metabolic Syndrome in Children and Adolescents: Diagnostic Criteria, Therapeutic Options and Perspectives. Curr Obes Rep. 2019; 8(4): 472479.

31 Denzer C, Thiere D, Muche R, et al. Gender-specific prevalences of fatty liver in obese children and adolescents: roles of body fat distribution, sex steroids, and insulin resistance. $J$ Clin Endocrinol Metab. 2009; 94(10): 3872-3881.

32 De Nooijer A, Vreugdenhil A, Karnebeek K, et al. A narrative review of factors associated with the development and progression of non-alcoholic fatty liver disease. GastroHep. 2019; 1(4): 180-191.

33 Buzzetti E, Pinzani M, Tsochatzis EA. The multiple-hit pathogenesis of non-alcoholic fatty liver disease (NAFLD). Metabolism. 2016; 65(8): 1038-1048.

34 Zhao K, Ju H, Wang $\mathrm{H}$. Metabolic characteristics of obese children with fatty liver: A STROBE-compliant article. Medicine (Baltimore). 2019; 98(16): e14939-e14939.

35 Ullah R, Rauf N, Nabi G, et al. Role of Nutrition in the Pathogenesis and Prevention of Non-alcoholic Fatty Liver Disease: Recent Updates. Int J Biol Sci. 2019; 15(2): 265-276.

36 Aron-Wisnewsky J, Clement K, Pépin JL. Nonalcoholic fatty liver disease and obstructive sleep apnea. Metabolism. 2016; 65(8): 1124-1135.

37 Sookoian S, Pirola CJ. Systematic review with meta-analysis: the significance of histological disease severity in lean patients with nonalcoholic fatty liver disease. Aliment Pharmacol Ther. 2018; 47(1): 16-25.

38 Wang AY, Dhaliwal J, Mouzaki M. Lean non-alcoholic fatty liver disease. Clin Nutr. 2019; 38(3): 975-981.

39 Koliaki C, Szendroedi J, Kaul K, et al. Adaptation of hepatic mitochondrial function in humans with non-alcoholic fatty liver is lost in steatohepatitis. Cell Metab. 2015; 21(5): 739746. 
40 Afolabi PR, Scorletti E, Smith DE, et al. The characterisation of hepatic mitochondrial function in patients with non-alcoholic fatty liver disease (NAFLD) using the 13Cketoisocaproate breath test. J Breath Res. 2018; 12(4): 046002.

41 Belei O, Olariu L, Dobrescu A, et al. The relationship between non-alcoholic fatty liver disease and small intestinal bacterial overgrowth among overweight and obese children and adolescents. J Pediatr Endocrinol Metab. 2017; 30: 1161-1168.

42 Schwimmer JB, Johnson JS, Angeles JE, et al. Microbiome Signatures Associated With Steatohepatitis and Moderate to Severe Fibrosis in Children With Nonalcoholic Fatty Liver Disease. Gastroenterology. 2019;v157(34): 1109-1122.

43 Schwimmer JB, Celedon MA, Lavine JE, et al. Heritability of Nonalcoholic Fatty Liver Disease. Gastroenterology. 2009; 136: 1585-1592.

44 Marzuillo P, Miraglia del Giudice E, Santoro N. Pediatric fatty liver disease: Role of ethnicity and genetics. World J Gastroenterol. 2014; 20(23): 7347-7355.

45 Zusi C, Mantovani A, Olivieri F, et al. Contribution of a genetic risk score to clinical prediction of hepatic steatosis in obese children and adolescents. Dig Liver Dis. 2019; 51(11): 1586-1592.

46 Romeo S, Kozlitina J, Xing C, et al. Genetic variation in PNPLA3 confers susceptibility to nonalcoholic fatty liver disease. Nat Genet. 2008; 40(12): 1461-1465.

47 Wesolowski SR, Kasmi KCE, Jonscher KR, et al. Developmental origins of NAFLD: a womb with a clue. Nat Rev Gastroenterol Hepatol. 2017; 14: 81-96.

48 Bellatorre A, Scherzinger A, Stamm E, et al. Fetal Overnutrition and Adolescent Hepatic Fat Fraction: The Exploring Perinatal Outcomes in Children Study. J Pediatr. 2018; 192: 165170.e1.

49 Soderborg TK, Clark SE, Mulligan CE, et al. The gut microbiota in infants of obese mothers increases inflammation and susceptibility to NAFLD. Nat Commun. 2018; 9(1): 4462.

50 Soderborg TK, Friedman JE. Imbalance in gut microbes from babies born to obese mothers increases gut permeability and myeloid cell adaptations that provoke obesity and NAFLD. Microb Cell. 2019; 6(1): 102-104.

51 Newton KP, Feldman HS, Chambers CD, et al. Low and High Birth Weights Are Risk Factors for Nonalcoholic Fatty Liver Disease in Children. J Pediatr. 2017; 187: 141-146.e1.

52 Li X, Shi X, Hou Y, et al. Paternal hyperglycemia induces transgenerational inheritance of susceptibility to hepatic steatosis in rats involving altered methylation on Ppara promoter. Biochim Biophys Acta Mol Basis Dis. 2019; 1865(1): 147-160. 
53 Treviño LS, Katz TA. Endocrine Disruptors and Developmental Origins of Nonalcoholic Fatty Liver Disease. Endocrinology. 2018; 159: 20-31.

54 Mann JP, Tang GY, Nobili V, et al. Evaluations of Lifestyle, Dietary, and Pharmacologic Treatments for Pediatric Nonalcoholic Fatty Liver Disease: A Systematic Review. Clin Gastroenterol Hepatol. 2019; 17(8): 1457-1476.e7.

55 Schwimmer JB, Ugalde-Nicalo P, Welsh JA, et al. Effect of a Low Free Sugar Diet vs Usual Diet on Nonalcoholic Fatty Liver Disease in Adolescent Boys: A Randomized Clinical Trial. JAMA. 2019; 321(3): 256-265.

56 Africa JA, Newton KP, Schwimmer JB. Lifestyle Interventions Including Nutrition, Exercise, and Supplements for Nonalcoholic Fatty Liver Disease in Children. Dig Dis Sci. 2016; 61(5): 1375-1386.

57 Gibson PS, Lang S, Dhawan A, et al. Systematic Review: Nutrition and Physical Activity in the Management of Paediatric Nonalcoholic Fatty Liver Disease. J Pediatric Gastroenterol Nutr. 2017; 65(2): 141-149.

58 Sundaram SS, Halbower AC, Klawitter J, et al. Obstructive Sleep Apnea and Chronic Intermittent Hypoxia Improves the Severity of Nonalcoholic Fatty Liver Disease in Children. J Pediatr. 2018; 198: 67-75.e1.

59 Yodoshi T, Orkin S, Arce-Clachar AC, et al. Vitamin D deficiency: prevalence and association with liver disease severity in pediatric nonalcoholic fatty liver disease. Eur J Clin Nutr. 2019. doi: 10.1038/s41430-019-0493-y. [Epub ahead of print]

60 Karaivazoglou K, Kalogeropoulou M, Assimakopoulos S, et al. Psychosocial Issues in Pediatric Nonalcoholic Fatty Liver Disease. Psychosomatics. 2019; 60(1): 10-17.

61 Lavine JE, Schwimmer JB, Natta MLV, et al. Effect of Vitamin E or Metformin for Treatment of Nonalcoholic Fatty Liver Disease in Children and Adolescents: The TONIC Randomized Controlled Trial. JAMA. 2011; 305: 1659-1668.

62 McDonagh MS, Selph S, Ozpinar A, et al. Systematic Review of the Benefits and Risks of Metformin in Treating Obesity in Children Aged 18 Years and Younger. JAMA Pediatr. 2014; 168(2): 178-184.

63 Amanullah I, Khan YH, Anwar I, et al. Effect of vitamin E in non-alcoholic fatty liver disease: a systematic review and meta-analysis of randomised controlled trials. Postgrad Med J. 2019; 95(1129): 601-611.

64 National Institute for Health and Care Excellence. Non-alcoholic fatty liver disease (NAFLD): assessment and management. [NG49]. 2016. London: National Institute for Health and Care Excellence. 
65 Nobili V, Alisi A, Mosca A, et al. The Antioxidant Effects of Hydroxytyrosol and Vitamin E on Pediatric Nonalcoholic Fatty Liver Disease, in a Clinical Trial: A New Treatment? Antiox Redox Signal. 2019; 31(2): 127-133.

66 Schwimmer JB, Lavine JE, Wilson LA, et al. In Children With Nonalcoholic Fatty Liver Disease, Cysteamine Bitartrate Delayed Release Improves Liver Enzymes but Does Not Reduce Disease Activity Scores. Gastroenterology. 2016; 151(6): 1141-1154.e9.

67 Nobili V, Alisi A, Musso G, et al. Omega-3 fatty acids: Mechanisms of benefit and therapeutic effects in pediatric and adult NAFLD. Crit Rev Clin Lab Sci. 2016; 53(2): 106-120.

68 Scorletti E, Byrne CD. Omega-3 fatty acids and non-alcoholic fatty liver disease: Evidence of efficacy and mechanism of action. Mol Aspects Med. 2018; 64: 135-146.

69 Nobili V, Putignani L, Mosca A, et al. Bifidobacteria and lactobacilli in the gut microbiome of children with non-alcoholic fatty liver disease: which strains act as health players? Arch Med Sci. 2018; 14(1): 81-87.

70 Beamish AJ, Reinehr T. Should bariatric surgery be performed in adolescents? Eur J Endocrinol. 2017; 176: D1-D15.

71 Nobili V, Vajro P, Dezsofi A, et al. Indications and Limitations of Bariatric Intervention in Severely Obese Children and Adolescents With and Without Nonalcoholic Steatohepatitis: ESPGHAN Hepatology Committee Position Statement. J Pediatr Gastroenterol Nutr. 2015; 60(4): 550-561.

72 Vos MB, Jin R, Konomi JV, et al. A randomized, controlled, crossover pilot study of losartan for pediatric nonalcoholic fatty liver disease. Pilot Feasibility Stud. 2018; 4: 109.

73 Ratziu V, Harrison SA, Francque S, et al. Elafibranor, an agonist of the peroxisome proliferator-activated receptor- $\alpha$ and $-\delta$, induces resolution of non-alcoholic steatohepatitis without fibrosis worsening. Gastroenterology. 2016; 150(5): 1147-1159 